\title{
Performance of Powerline-Controlled Luminaire Final Report
}

\author{
Francis Rubinstein \\ Lawrence Berkeley National Laboratory \\ Pete Pettler \\ Joel Snook \\ Erik Engelking \\ Vistron \\ Sila Kiliccote \\ Lawrence Berkeley National Laboratory
}

This work was supported by the Assistant Secretary for Energy Efficiency and Renewable Energy, Building Technologies Program, of the U.S. Department of Energy under Contract No. DE-AC03-76SF00098. 


\section{Table of Contents}

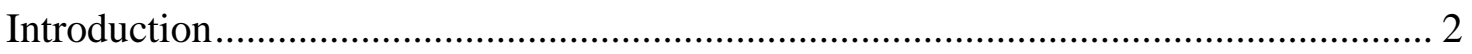

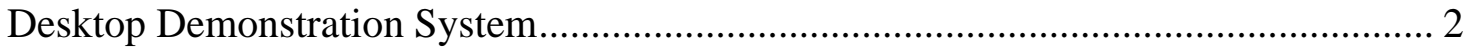

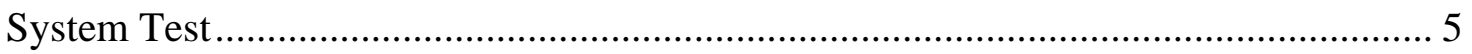

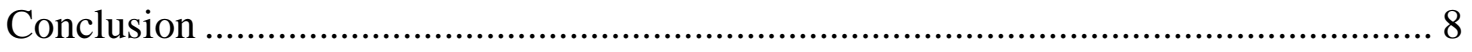

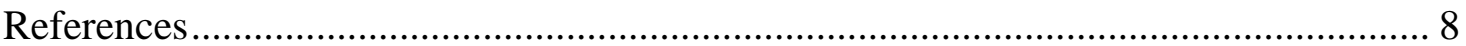




\section{Introduction}

In previous work, LBNL with Vistron Corp, developed an innovative lighting control system using a communications technology called Phase Cut Carrier (PCC). This report describes the performance of the desktop demonstration system that was developed to test this new controls concept. More detailed information on this project is given in [1]. This report is in fulfillment of deliverable \#1 "Report on Performance of Powerline-carrier Controlled Luminaire” from the FY2004 DOE Work Plan.

\section{Desktop Demonstration System}

The key components of the desktop demonstration system are given in the following figures.

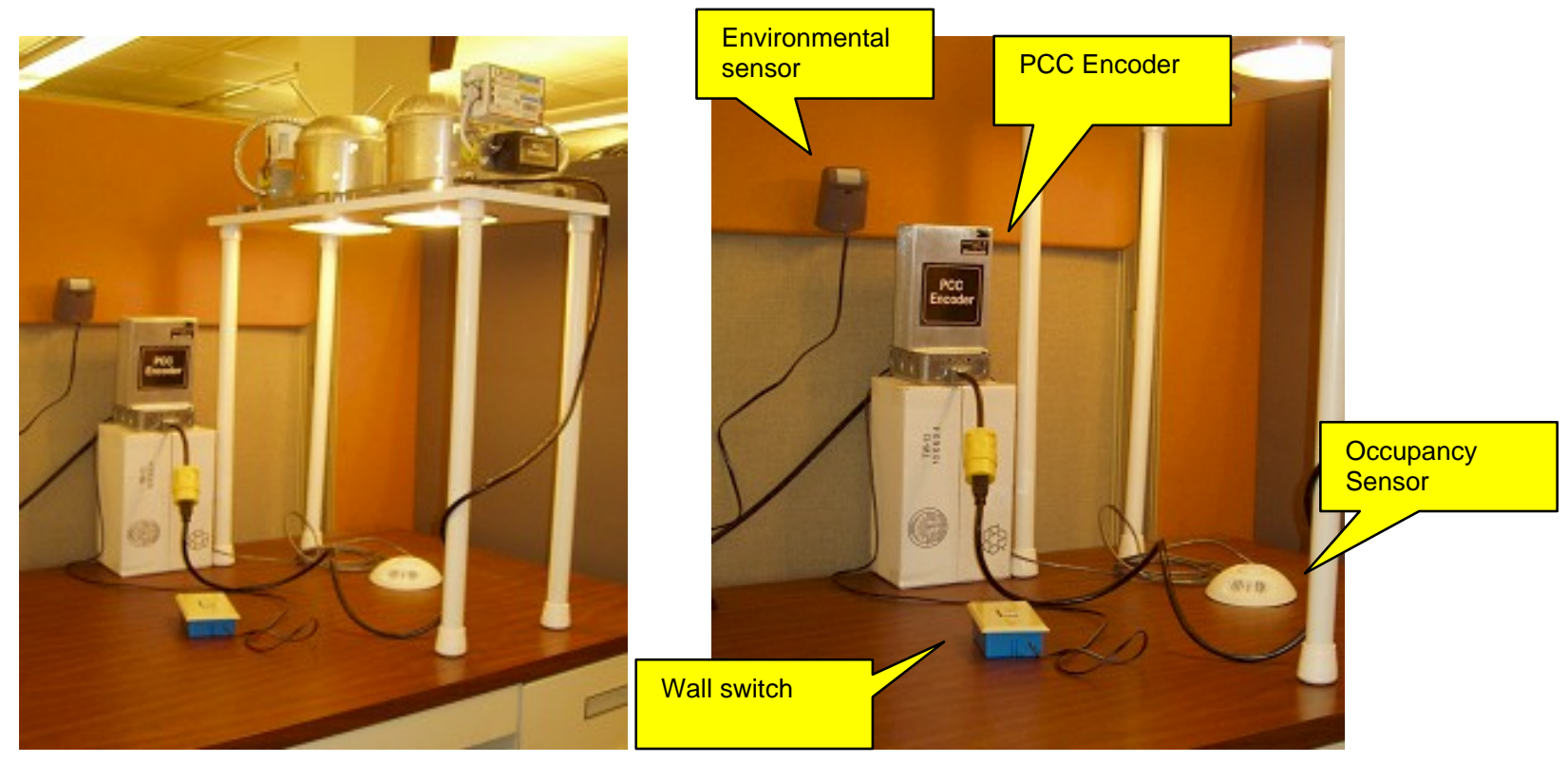

Figure 1. Entire desktop demonstration of the Phase Cut Carrier system.

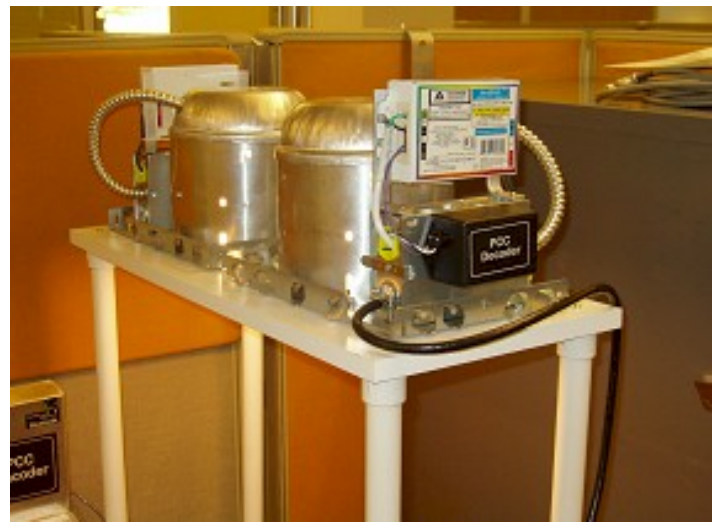

Figure 3. View of the lights at the top of the desktop demonstration of the Phase Cut Carrier system.
Figure 2. Closer view of the entire desktop demonstration of the Phase Cut Carrier system.

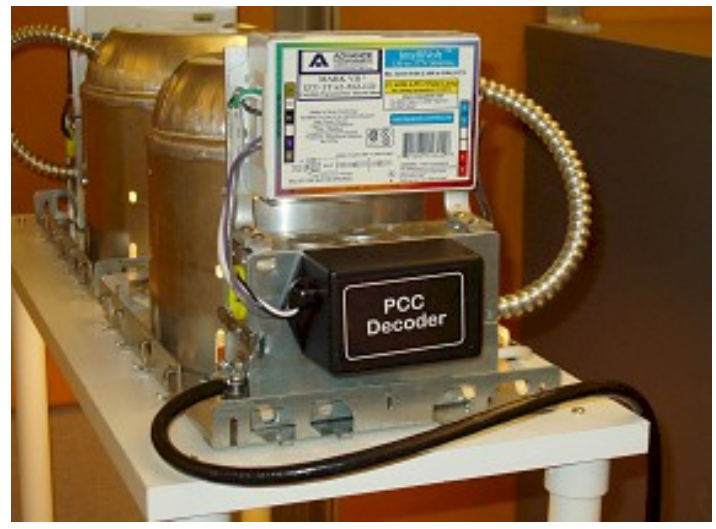

Figure 4. Closeup of the PCC Decoder and the electronic dimming ballast (0-10 VDC) controlled by the system. 


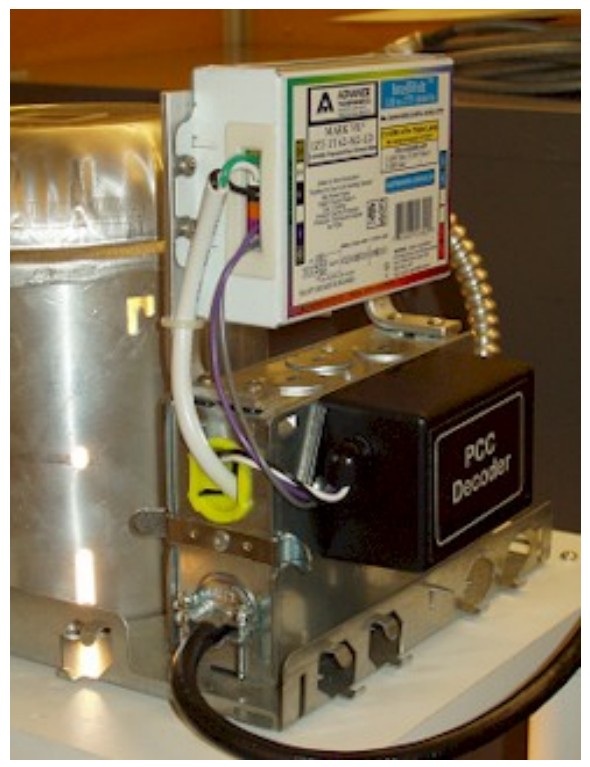

Figure 5. Closeup shot of the PCC Decoder showing the electrical connections between the decoder and the ballast.

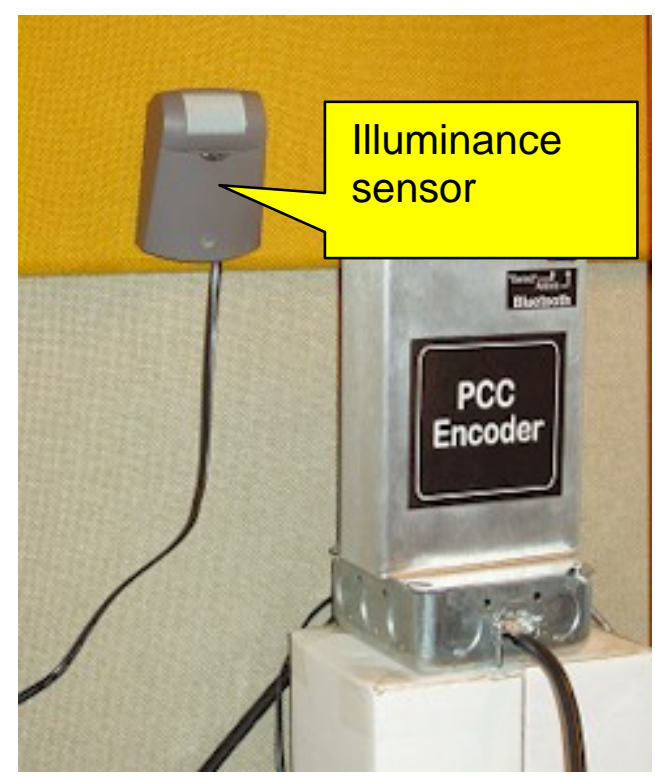

Figure 6. Closer view of the PCC Encoder and attached junction box (which collectively form the "intelligent junction box"). Also shown to the left is the environmental sensor that is attached to the wall. Note the location of the illuminance sensor within the environmental sensor.

The objective of this task was to install the innovative control system in a daylit office and measure the instantaneous power consumption, task light levels and energy savings over several representative days.

Prior to testing the onsite performance of the PCC control system, the following data points are studied. Their relevance to the control system analysis is considered below:

\section{Instantaneous Wattage}

Table 1 below shows the instantaneous wattage consumed at eight dimming levels with the corresponding percentage of light output. At minimum light output (25\%), the system draws $36.7 \%$ of maximum power. This loss in efficacy at full dim is typical of fluorescent lighting systems. The specific locations of the steps in light output are under firmware control. We have elected to make the steps evenly spaced with the minimum level set to 25\% light output. Different ranges, step sizes, and step spacing are easily changed by changing the firmware in the PCC Encoder and Decoder. In production, the manufacturer of the ballast would dictate the values of these parameters.

Table 1. Instantaneous wattage consumed at eight dimming levels.

\begin{tabular}{|c|c|c|}
\hline Dimming Level & $\begin{array}{c}\text { Instantaneous } \\
\text { Power (watts) }\end{array}$ & $\begin{array}{c}\text { \% Light } \\
\text { Output }\end{array}$ \\
\hline 0 & 18 & 25 \\
\hline 1 & 21 & 36 \\
\hline 2 & 25 & 46 \\
\hline
\end{tabular}




\begin{tabular}{|c|c|c|}
\hline 3 & 28 & 57 \\
\hline 4 & 32 & 67 \\
\hline 5 & 38 & 79 \\
\hline 6 & 42 & 89 \\
\hline 7 & 49 & 100 \\
\hline
\end{tabular}

\section{Elapsed Energy Consumption}

The accumulated energy consumption (in watt-hours) of the lighting circuit fed through the Encoder is indicated in the display. It appears that this annunciator is not properly calibrated for our setup as the accumulated energy consumption was too high by at least a factor of 5 . This may be because some parameters are not set correctly.

\section{Dimming Level}

The value of this field varies from 0 to 7 (see table above) and corresponds with the instantaneous wattage fluctuations. Even though the absolute values of the instant wattage are not verified, the synchronous movement of these two values show that the system's data collection and recording capability are working.

\section{Occupancy Sensor Status}

This occupancy sensor is connected directly to the PCC Encoder. It is intended as a room-based detector. The override placed during the duration of testing forces the value of the data field to "on". Therefore, the collected data shows that the value of this field is always on. This verifies two issues: 1) the occupancy sensor can be overridden; 2) during the period when the tests were conducted, the occupancy sensor was not an operational part of this system's control scheme.

\section{Environmental Sensor}

The three following components, specifically illuminance sensor, workstation occupancy detection, and temperature, are all integrated into one device.

\section{Illuminance Sensor}

To test the linearity of the illuminance sensor portion of the environmental sensor, a handheld Minolta illuminance meter is used. The dimming levels are varied in a nondaylit environment and readings are taken at the same point with both the illuminance meter and the multi-sensor. The results are summarized below:

Table 2. Results of illumance measurements with handheld meter.

\begin{tabular}{|c|c|c|c|c|}
\hline Multi Sensor (lux) & Minolta (lux) & $\begin{array}{l}\text { Normalized } \\
\text { Multi sensor }\end{array}$ & $\begin{array}{l}\text { Normalized } \\
\text { Minolta }\end{array}$ & \multicolumn{1}{|l|}{ Error } \\
\hline 128 & 163 & 0.267 & 0.267 & 0.00 \\
\hline 182 & 234 & 0.379 & 0.384 & 0.00 \\
\hline 243 & 313 & 0.506 & 0.513 & 0.01 \\
\hline 294 & 377 & 0.613 & 0.618 & 0.01 \\
\hline 358 & 455 & 0.746 & 0.746 & 0.00 \\
\hline 397 & 510 & 0.827 & 0.836 & 0.01 \\
\hline 435 & 560 & 0.906 & 0.918 & 0.01 \\
\hline
\end{tabular}


480

610

1.000

1.000

0.00

The normalized values of the multi sensor and the handheld Minolta match closely with 0-0.01 error range. The results show that the sensor responds linearly to changes in light output as expected.

\section{Workstation Occupancy Sensor}

The motion sensor is functioning as specified. The time delay adjustment was 30 seconds during the testing period. The motion detector was temporarily bypassed so it did not interfere with the control system tests.

\section{Temperature Sensor}

A Radio Shack digital thermometer was used to compare the temperature reading of the multi-sensor. Spot measurements concur with multi-sensor readings.

\section{Date and Time Stamp}

The date and time stamp values of the log file coincide with the date and time settings of the computer.

\section{System Test}

The desktop demonstration unit was set up at LBNL in an unoccupied daylit office between July $21^{\text {st }}$ and $30^{\text {th }}$ for testing its overall performance as well as the automatic response to daylight. During this period, the occupancy sensor was disabled and the system was set to run on control mode. The target light level was set to 500 lux with maximum deviation from this target illuminance set to +/-40 lux. The PCC system's capability as a data acquisition system was exploited to simply data collection. A log file, called "status.log", is initiated by the PCC software and collected the following information:

- Instantaneous wattage

- Average wattage

- The dimming level recorded as a value between 0 and 7

- Occupancy sensor status: Since this sensor was overridden, sensor data field yields “occupied” during the test period.

- Illuminance level at the multi sensor

- Motion sensor status: This sensor's reading was bypassed. Therefore, although it continued to collect the occupancy sensor information, this information was not used.

- Room temperature recorded in Celsius

- Time of day and date

The electrical information collected by the desktop system was used to judge its performance. 


\section{Evaluation of Daylight Responsive Element}

In this test, the PCC control system was evaluated in terms of its ability to respond to the changing daylight conditions and to keep the illuminance at the environmental sensor constant within prescribed limits.

The PCC system's response to variation in indoor illuminance

The PCC system can be set to save energy by adjusting the electrical light component, as available daylight increases. To confirm this, the system was set up for automatic control. Using the data logging feature of the PCC system, data was collected in 1-minute intervals. To enable automatic daylight control, the user expands the view of the user interface, checks the "Disable Automatic Controls" box and sets the target illuminance to the desired level. For our test, we used 500 lux that prior measurements in the test room had showed was roughly equal to the maximum daylight level alone. This meant that the room would have enough daylight that the test would be meaningful. Three days of usable data was extracted from the data collected over 9 days. July 24, 25 and 26 were selected for analysis because the motion sensor recorded minimum occupancy for those dates. The power, illuminance and temperature profiles for July 24, 25, and 26 are displayed in Figures 7, 8, and 9.

The data confirms that the PCC control system is effective in responding to the variations in the indoor illuminance. The systems starts dimming to maintain 500 lux as the daylight contribution increases. The dimming profile is clean and free of evidences for hunting and spikes. For example, on the $24^{\text {th }}$, starting at 8:43 am and around 500 lux, the dimming of the system is utilized constantly for 2 hours and 20 minutes. The length of the dimming period iterates the fact that when daylight availability of a space is limited, dimming capability plays a bigger role in the design of lighting systems. 


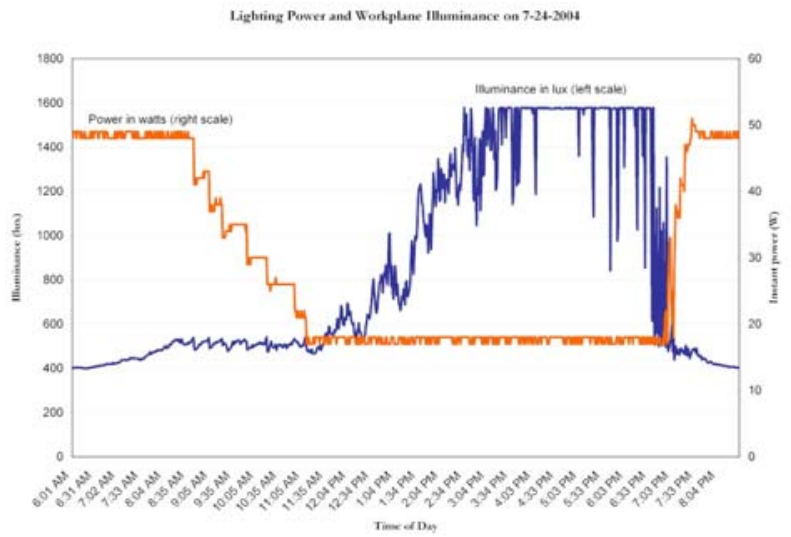

Figure 7A. Lighting power and workplane illuminance July 24, 2004.

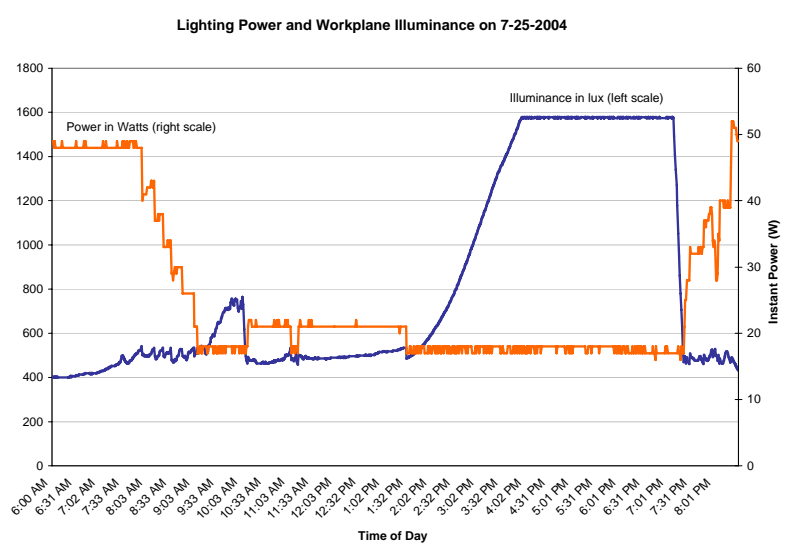

Figure 8A. Lighting power and workplane illuminance July 25, 2004.

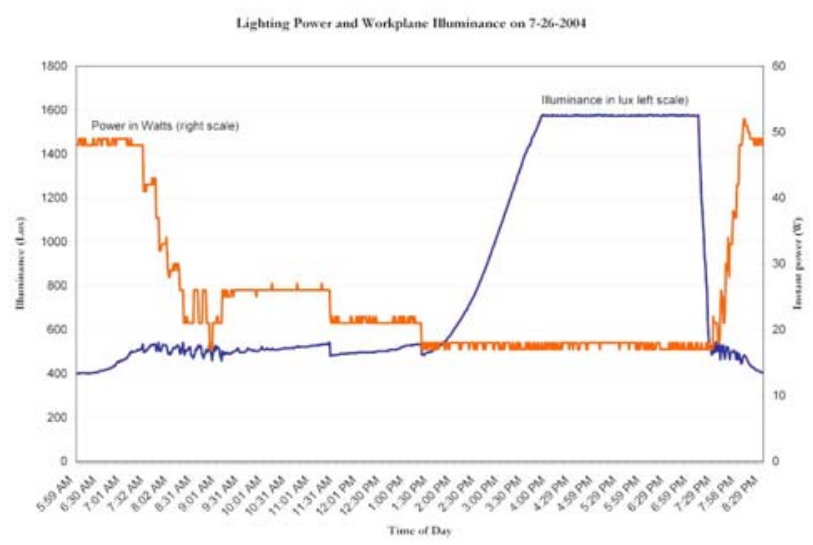

Figure 9A. Lighting power and workplane illuminance July 26, 2004.

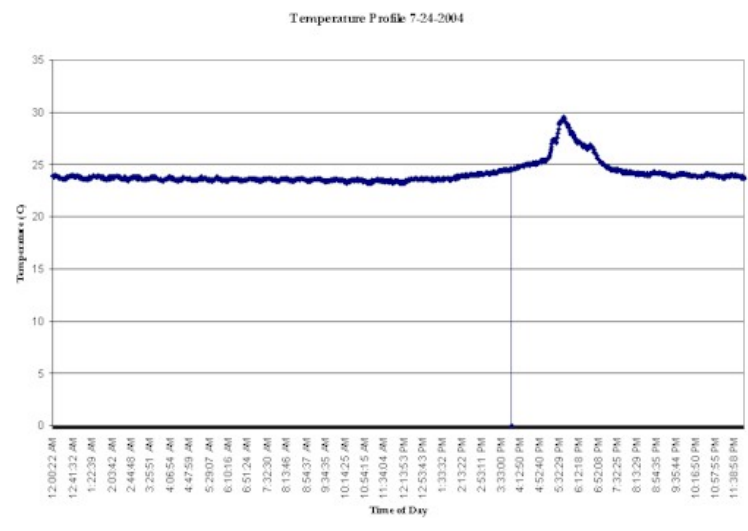

Figure 7B. Temperature at the workplane, as measured by environmental sensor, for July 24, 2004

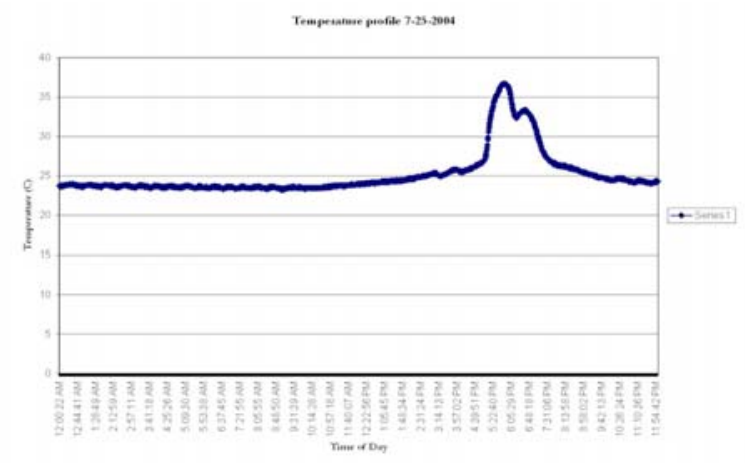

Figure 8B. Temperature at the workplane, as measured by environmental sensor, for July 25, 2004

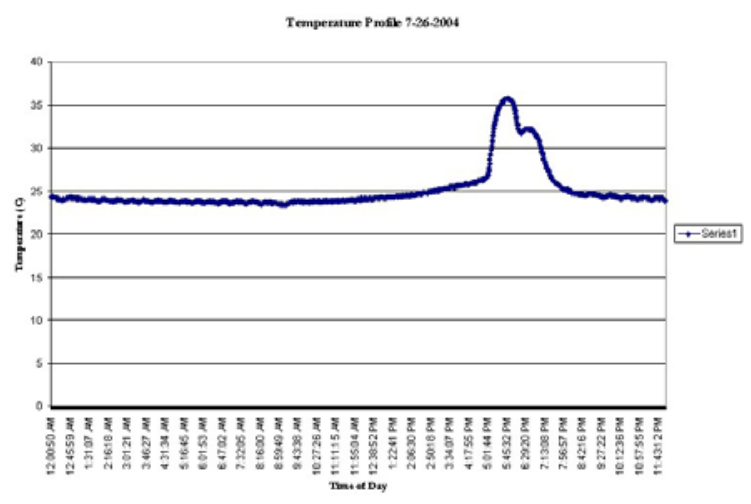

Figure 9B. Temperature at the workplane, as measured by environmental sensor, for July 26, 2004 
The PCC system's ability to keep within the target illuminance

Figures 7, 8 and 9 demonstrate that the system is successful in maintaining the 500 lux target illuminance on the work surface where the multi-sensor is located. The system's response at the end of the day when the multi sensor moves from direct sunlight to sunset as evidenced by the temperature sensor, is even more sudden and a better test. Even when the conditions change abruptly, the system is able to track and respond to these changes as designed.

We used the data collected on the log file to calculate the energy savings for each day of the test. Using 6 AM to $6 \mathrm{PM}$ as the hours of operation, the energy savings are 44, 49, and $49 \%$ for July 24,25 , and 26 , respectively. This shows conclusively that the daylight responsive portion of the control system works correctly, saves significant energy, and reduces light power most during utility peak hours (12 noon - 6 PM).

\section{Conclusion}

The powerline carrier controlled luminaire was tested in an office at LBNL and was found to be able to track changes in daylighting with good accuracy and impressive energy savings results.

\section{References}

Rubinstein, Pettler, Snook, "Retrofit Fluorescent Dimming with Integrated Lighting Controls”, Final Report, prepared for the California Energy Commission Public Interest Energy Research (PIER) Program, December, 2004. 